\title{
3,3'-Diindolylmethane suppresses the growth of gastric cancer cells via activation of the Hippo signaling pathway
}

\author{
XIU JUAN LI ${ }^{1}$, EUN SUNG PARK ${ }^{2}$, MAN HEE PARK $^{3}$ and SOO MI KIM ${ }^{1}$ \\ ${ }^{1}$ Department of Physiology, Institute for Medical Sciences, Chonbuk National University Medical School, Jeonju; \\ ${ }^{2}$ Institute for Medical Convergence, Yonsei University College of Medicine, Seoul; \\ ${ }^{3}$ Catholic University of Pusan, Busan, Republic of Korea
}

Received July 22, 2013; Accepted August 23, 2013

DOI: $10.3892 /$ or.2013.2717

\begin{abstract}
Recent studies have revealed that 3,3-diindolylmethane (DIM) has antitumor effects in both in vivo and in vitro tumor models. However, the biological function of DIM in human gastric cancer cells is unknown. Genetic and biological studies have confirmed the importance of the novel Hippo tumor-suppressor pathway in regulating cell proliferation, apoptosis, organ size and tumorigenesis in mammals. Thus, the purpose of this study was to investigate the cytotoxic effects of DIM in human gastric cancer cells and to elucidate whether DIM induces cell death by activating the Hippo signaling pathway. Two human gastric cancer cell lines (SNU-1 and SNU-484) were used to investigate the DIM response. DIM significantly inhibited the proliferation of human gastric cancer cells in a dose-dependent manner. The percentage of G1 phase cells increased $24 \mathrm{~h}$ following DIM treatment. DIM reduced CDK2, CDK4, CDK6 and cyclin D1 protein levels, while increasing p53 protein levels. DIM induced the levels of cleaved poly(ADP-ribose) polymerase, cleaved-caspase-9, and diminished pro-caspase-3 protein production. In addition, DIM increased pLATS1, Mob1, pMob1, pYAP and Ras association domain family 1 (RASSF1) protein levels and reduced Yap protein production levels. DIM stimulated the binding of RASSF1 with the Mst1/2-LATS1-Mob1 complex, promoting an active Hippo signaling pathway and favoring YAP phosphorylation (pYAP) that inactivates cell proliferation. Furthermore, DIM inhibited the growth of human gastric tumors in a xenograft mouse model. These results indicate that DIM suppresses the growth of gastric cancer cells by activating the Hippo signaling pathway.
\end{abstract}

Correspondence to: Dr Soo Mi Kim, Department of Physiology, Chonbuk National University Medical School, 2-20 Keum-AmDong-San, Jeonju 561-180, Republic of Korea

E-mail: soomikim@jbnu.ac.kr

Key words: 3,3'-diindolylmethane, apoptosis, Hippo signaling pathway

\section{Introduction}

Gastric cancer is one of the most lethal cancers in Asia and causes approximately 800,000 deaths worldwide each year, making it the second leading cause of cancer-related mortality in the world (1-3). Gastric cancer is thought to arise from a sequence of multistep processes involving a variety of genetic events $(4,5)$. Despite advances in multimodality treatments including targeted therapies, the clinical outcome of gastric cancer still remains poor due to de novo or acquisition of chemoresistance during therapy (6-8). Moreover, the overall 5 -year survival rate is less than $35 \%$ due to the high rate of relapse after gastrectomy (9). Thus, understanding the molecular characteristics of gastric cancer and the development of chemoresistance is urgently needed in order to improve the clinical outcome of gastric cancer patients and to develop more effective treatment strategies.

The Hippo signaling pathway, also known as the Salvador-Warts-Hippo pathway, was originally discovered in Drosophila melanogaster (10-12). The pathway core components in the fly, including Hippo, Sav, Wts, Yki and Mats, are highly conserved in mammals as Mst1/2, WW45, LATS1/2, YAP and Mob1, respectively $(11,13,14)$. Recently, several studies have clearly shown the role of the Hippo pathway in controlling organ size and other biological processes including cell fate determination, mitosis and pluripotency $(11,15,16)$. YAP is a key effector protein in the Hippo pathway and is negatively regulated by the Mst1/2-LATS1/2-Mob1 complex through direct phosphorylation (11). The phosphorylation of YAP (pYAP) inhibits cell proliferation; therefore, impairment of the Hippo signaling pathway has been implicated in many human cancers including gastric cancer (17-22).

Natural products from vegetables are well acknowledged for their possible roles as chemopreventive agents (23). 3,3'-Diindolylmethane (DIM) is a principal product converted from the bioactive phytochemical indole-3-carbinol (I3C) that is found in abundance in cruciferous vegetables (24). I3C is chemically unstable in aqueous environments and is rapidly converted to DIM, which is a major condensation product in the stomach (25-27). DIM is the predominant bioactive compound in plasma (27), and recent studies have shown that DIM has anticancer effects in both in vivo and in vitro models of various types of cancers (28-37). However, the biological function of 
DIM, and its possible use as an antitumor agent for human gastric cancer, is unknown. Several studies have revealed the importance of the novel Hippo tumor-suppressor pathway in regulating cell proliferation, apoptosis and tumorigenesis in gastric cancer. Therefore, the purpose of the present study was to investigate the biological function of DIM in human gastric cancer cells and to elucidate whether DIM regulates the Hippo signaling pathway. Our results demonstrated that DIM activates the Hippo signaling pathway in sensitizing gastric cancer cells in vitro and inhibits gastric cancer tumors in vivo.

\section{Materials and methods}

Cell culture and reagents. The human gastric cancer cell lines SNU-1 and SNU-484 were purchased from the Korean Cell Line Bank (Seoul National University, Korea). Cancer cells were maintained in RPMI-1640 medium supplemented with $10 \%$ fetal bovine serum (FBS), $100 \mathrm{mg} / \mathrm{ml}$ streptomycin, and $100 \mathrm{IU} / \mathrm{ml}$ penicillin (all from Gibco-BRL, Grand Island, NY, USA) as a monolayer in 100-mm dishes (BD Biosciences, Rockville, MD, USA) under standard conditions at $37^{\circ} \mathrm{C}$ in a $5 \%$ $\mathrm{CO}_{2}$ humidified atmosphere. All experiments were performed with the cells at $60-80 \%$ confluence. DIM was purchased from LKT Laboratories (St. Paul, MN, USA). The following antibodies were obtained from the following commercial sources: cyclin D1, CDK4, CDK6, p27, cleaved-caspase-9, pro-caspase-3, cleaved-poly(ADP-ribose) polymerase (PARP), Mst1, Mst2, LATS1, pLATS1, Mob1, pMob1, Sav1, YAP, pYAP, Akt, pAkt (Cell Signaling Technology, Inc., Beverly, MA, USA); CDK2 and Ras association domain family 1 (RASSF1) antibody (Santa Cruz Biotechnology, Inc., Santa Cruz, CA, USA).

Cell growth inhibition by MTT assay. The effect of DIM on cell proliferation was determined by the 3-(4,5-dimethylthiazol-2-yl)-2,5-diphenyltetrazolium bromide (MTT) assay as described previously (38). Briefly, cells were plated in 96-well plates (SPL, Seoul, Korea) at $1 \times 10^{4}$ cells/well. After $24 \mathrm{~h}$ of incubation under standard conditions, the cells were treated with the indicated DIM concentration for $72 \mathrm{~h}$. Cell viability was assessed by a scanning multi-well spectrophotometer (SpectraMAX 340; Molecular Devices Co., Sunnyvale, CA, USA).

Soft agar colony formation assay. The bottom layer of soft agar $(1 \%)$ was prepared in a 6-well plate, and the top layer $(0.7 \%)$ was prepared with $5 \times 10^{4}$ SNU-1 or SNU-484 cells/ well in a single-cell suspension. The cells were divided into 2 groups: i) the control group (blank group) and ii) the experimental group (DIM, $100 \mu \mathrm{M}$ ). The cells were cultured in an incubator at $37^{\circ} \mathrm{C}$ with $5 \% \mathrm{CO}_{2}$ for 2 weeks and observed for colony formation by microscopy. Colonies of $>30$ cells were counted, and the experiments were repeated in triplicate.

Cell cycle analysis. To determine the effect of DIM on the cell cycle, cells were incubated in 100-mm dishes and were treated for $24 \mathrm{~h}$ with various DIM concentrations. Subsequently, cells were washed with PBS and the nuclei were stained with propidium iodide (PI) (Sigma Chemical, St. Louis, MO, USA) as described previously $(28,38)$. The percentage of cells in the different cell cycle phases was measured with a FACstar flow cytometer (Becton-Dickinson, San Jose, CA, USA) and analyzed using Becton-Dickinson software (Lysis II and CellFit).

Western blot analysis. Briefly, the human gastric cancer cell lines SNU-1 and SNU-484 were plated and allowed to attach for $24 \mathrm{~h}$. DIM was added to the cell cultures at the indicated concentrations for $72 \mathrm{~h}$. Cells with or without DIM were harvested and suspended in lysis buffer (Intron Biotechnology, Korea). Extracts were incubated on ice for $10 \mathrm{~min}$ and centrifuged at $13,200 \mathrm{rpm}$ for $20 \mathrm{~min}$ at $4^{\circ} \mathrm{C}$. After centrifugation, the supernatant was collected and the protein concentration was determined using a BSA protein assay kit (Pierce Biotechnology, Inc., Rockford, IL, USA). Whole lysate was resolved on a SDS-PAGE gel and transferred to PVDF membranes (Bio-Rad, Hercules, CA, USA). Membranes were probed with the specific primary antibodies and then with peroxidase-conjugated secondary antibodies. The bands were visualized with the enhanced chemiluminescence kit (Amersham, Arlington Heights, IL, USA). The following antibodies were used: antibody against cyclin D1, CDK2, CDK4, CDK6, p53, cleaved-PARP, cleaved-caspase-9, pro-caspase-3, Mst1, Mst2, pLATS1, LATS1, Mob1, pMob1, Sav1, pYAP, YAP, pAkt, Akt, RASSF1, $\beta$-catenin, $p \beta$-catenin, $\beta$-actin and GAPDH.

Immunoprecipitation. Cells were scraped in PBS and suspended in lysis buffer (20 mM Tris- $\mathrm{HCl} \mathrm{pH} \mathrm{8,} 137 \mathrm{mM} \mathrm{NaCl}$, $10 \%$ glycerol, $1 \%$ Nonidet P-40 and 2 mM EDTA). Extracts were incubated on ice for $1 \mathrm{~h}$ and pelleted by centrifugation at $12,000 \mathrm{rpm}$ for $10 \mathrm{~min}$. Protein quantification was determined using a BSA protein assay kit. Whole lysates $(1.5 \mathrm{mg})$ were incubated with Protein G-Sepharose (Sigma) for $1 \mathrm{~h}$ at $4^{\circ} \mathrm{C}$ for pre-clearing and centrifuged $10,000 \mathrm{rpm}$ for $5 \mathrm{~min}$ at $4^{\circ} \mathrm{C}$. The supernatant was incubated with anti-RASSF1 at $4^{\circ} \mathrm{C}$ overnight. Antibody-antigen complexes were collected on Protein G-Sepharose. Immunoprecipitates were resolved by SDS-PAGE, transferred to PVDF membranes, and probed with the specific primary antibodies followed by peroxidase-conjugated secondary antibodies. The bands were visualized with the enhanced chemiluminescence kit. The following antibodies were used: antibody against Mst1, Mst2, LATS1, Mob1, Sav1 and GAPDH.

In vivo studies. Based on our in vitro results, we designed studies to determine the effects of DIM on xenografted human gastric tumors in nude mice. Animal experiments adhered to NIH guidelines (USA) and were performed under approval of the Institutional Animal Care and Use Committee of Chonbuk National University. Four-week-old female SPF/VAF immunodeficient mice were purchased from Orient Bio (Korea). The mice were allowed to acclimate to local conditions for 1 week prior to injection with cancer cells. Thirteen mice were injected subcutaneously (s.c.) into the right flank with $0.1 \mathrm{ml}$ Matrigel containing $3.5 \times 10^{6}$ human gastric cancer cells (SNU-484). The mice were randomized into 2 groups 1 week after tumor implantation: i) the untreated control group ( $\mathrm{n}=5$, DMSO in $50 \mu \mathrm{l}$ PBS daily) and ii) the DIM-treated group ( $\mathrm{n}=5,10 \mathrm{mg} /$ $\mathrm{kg}$ in $50 \mu \mathrm{l}$ PBS once daily). Gastric primary tumors were excised, and the final tumor volume was measured once every 
3 days using a caliper and calculated as (width) ${ }^{2} \mathrm{x}$ length $/ 2$. The experiment was terminated on day 39. Half of the tumor tissue was prepared for western blotting and the other half was snap frozen in liquid nitrogen and stored at $-80^{\circ} \mathrm{C}$.

Statistical analysis. In vitro experiments were repeated $>3$ times. The statistical significant difference between experimental groups and the control was determined by one-way ANOVA and then later compared among groups with an unpaired Student's t-test. Results are expressed as the means $\pm \mathrm{SE}$. A p-value $<0.05$ was considered to indicate a statistically significant result.

\section{Results}

DIM inhibits the proliferation of gastric cancer cells. The effects of DIM on human gastric adenocarcinoma cell growth were examined using SNU-1 and SNU-484 cells by treatment with various DIM concentrations for $72 \mathrm{~h}$. MTT assay analyses revealed that DIM significantly suppressed the proliferation of the cell lines in a dose-dependent manner, showing $>50 \%$ cell growth inhibition at $50 \mu \mathrm{M}$ in the two cell lines (Fig. 1A). The effects of DIM on SNU-1 and SNU-484 gastric cancer cell colony formation were evaluated using a soft agar cloning assay. SNU-1 and SNU-484 gastric cancer cells were cultured in medium with $100 \mu \mathrm{M}$ DIM for 2 weeks, and colony formation was observed by microscopy. As shown in Fig. 1B, DIM significantly inhibited colony formation of SNU-1 and SNU-484 cells when compared to the control. These results suggest that DIM significantly inhibits the growth of gastric cancer cells.

DIM induces cell cycle arrest in gastric cancer cells. Fluorescence-activated cell sorting (FACS) analysis was performed to characterize whether DIM regulates cell cycle progression in human gastric cancer cells. As shown in Fig. 2A, DIM treatment resulted in a significant increase in the proportion of the cell population in the G1 phase of the cell cycle at $24 \mathrm{~h}$ in SNU-1 and SNU-484 human gastric cancer cells. These results suggest that DIM induced G1 phase arrest in human gastric cancer cells in a dose-dependent manner. The effects of DIM on CDK2, CDK4, CDK6 and cyclin D1 protein levels were examined to identify the regulation of G1 cell cycle regulatory proteins in human gastric cancer cells. A $75-\mu \mathrm{M}$ DIM treatment in SNU-1 and SNU-484 cells downregulated the production of CDK2, CDK4, CDK6, cyclin D1 protein levels at $24 \mathrm{~h}$ (Fig. 2B). Since DNA damage checkpoints play a critical role in maintaining the integrity of the genome by arresting cell cycle progression in response to damaged or incompletely replicated DNA, and G1 checkpoint arrest in mammalian cells is mediated by the action of $\mathrm{p} 53$ protein, we also investigated the effects of DIM on the induction of the p53 protein level. A $75-\mu \mathrm{M}$ DIM treatment in SNU-1 and SNU-484 cells resulted in the upregulation of the 553 protein level leading to cell cycle arrest in the G1 phase (Fig. 2B). These results indicate that DIM induced G1 phase arrest in human gastric cancer cells through changes in the levels of cell cycle regulatory proteins.

DIM induces apoptosis in gastric cancer cells. As shown in Fig. 3A, DIM treatment in SNU-1 and SNU-484 gastric
A
DIM concentration $(\mu \mathrm{M})$

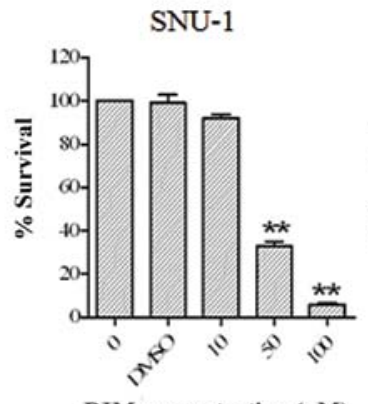

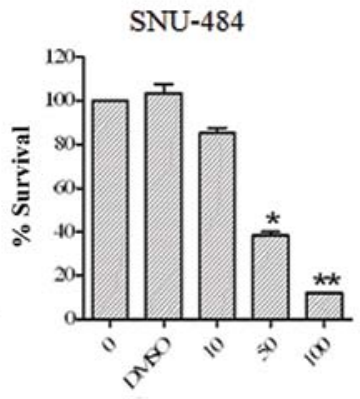

DIM concentration $(\mu \mathrm{M})$
B
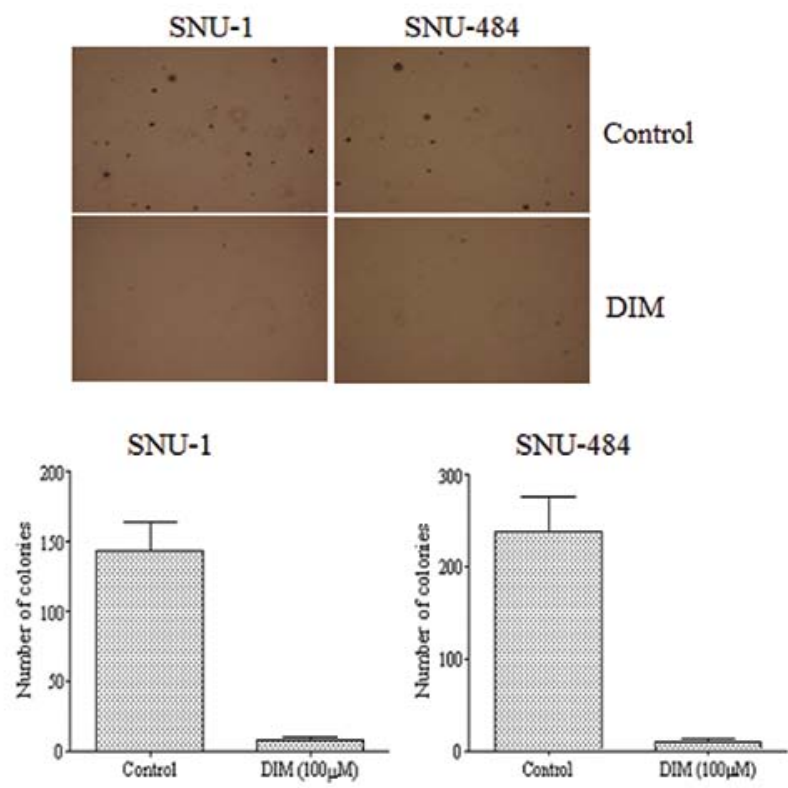

Figure 1. (A) Effect of DIM on growth inhibition in human gastric cancer cells. Logarithmically growing human gastric cancer cell lines (SNU-1 and SNU-484) were treated with various concentrations of DIM for $72 \mathrm{~h}$. Cell growth inhibition was assessed by MTT assay. Results are expressed as the percentage of control cells containing no DIM. Each point represents the means $\pm \mathrm{SE}$ of at least 3 independent experiments conducted in triplicate. * $\mathrm{p}<0.05$ and ${ }^{* *} \mathrm{p}<0.001$ when compared to the control. (B) Colony formation assay. DIM inhibits gastric cancer cell (SNU-1 and SNU-484) growth and proliferation. The assays were terminated at 2 weeks. DIM significantly reduced the colony formation ability in the gastric cancer cells. DIM, 3,3'-diindolylmethane.

cancer cells showed an increased accumulation of cells in the sub-G1 phase in a dose-dependent manner. To test whether DIM induces apoptotic cell death in gastric cancer cells, we further investigated the cleaved-caspase-9, cleaved-PARP, and pro-caspase-3 protein levels using western blot analysis. As shown in Fig. 3B, treatment with $100 \mu \mathrm{M}$ DIM for $72 \mathrm{~h}$ inhibited pro-caspase-3 protein levels and increased levels of the cleaved form of PARP and cleaved-caspase-9, hallmarks of apoptosis, in the SNU-1 and SNU-484 gastric cancer cells. These results indicate that DIM induced apoptotic cell death in human gastric cancer cells. Overall, these results support the notion that the observed decline in cell viability by DIM was in part due to cell cycle arrest and induction of apoptosis.

DIM activates the Hippo signaling pathway resulting in YAP inactivation. The Hippo signaling pathway is a tumor-suppressor signaling system that inhibits cell 
A

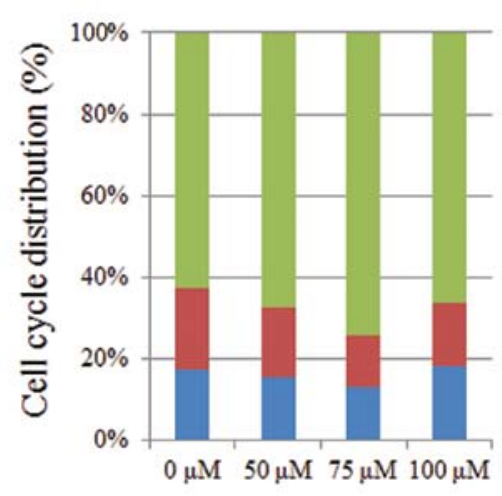

SNU-484

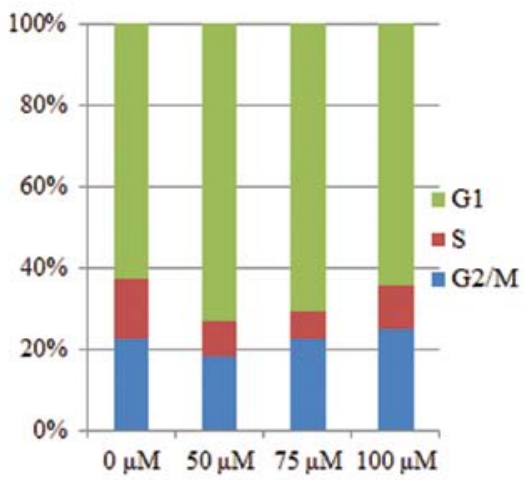

B
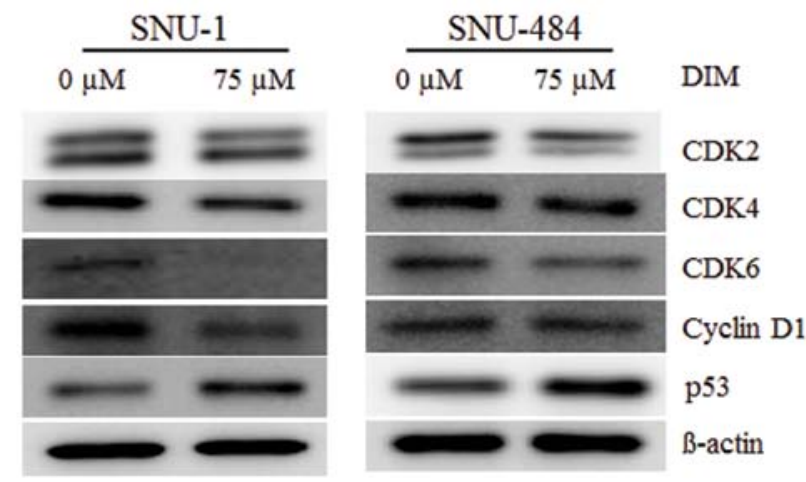

Figure 2. (A) Cell cycle analysis of human gastric cancer cells (SNU-1 and SNU-484) cultured with DIM. Cells were incubated with various concentrations of DIM for $24 \mathrm{~h}$. The control group represents gastric cancer cells that were not exposed to DIM. Cell cycle distribution was calculated as the percentage of cells in the G1, S, and G2/M phase. All experiments were performed at least 3 times. (B) Effects of DIM on cell cycle-related proteins in gastric cancer cell lines (SNU-1 and SNU-484). CDK2, CDK4, CDK6, cyclin D1, and p53 levels were assessed by western blotting. Cells were harvested at $24 \mathrm{~h}$. $\beta$-actin was used as an internal control. DIM, 3,3'-diindolylmethane.

A

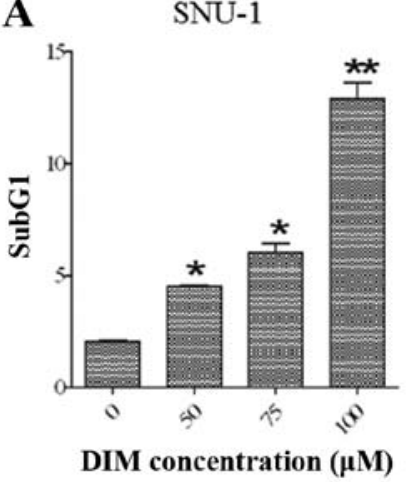

SNU-484

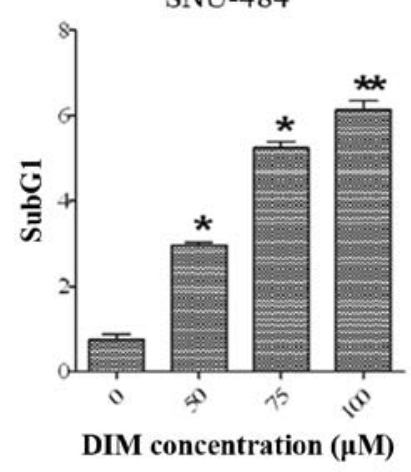

B
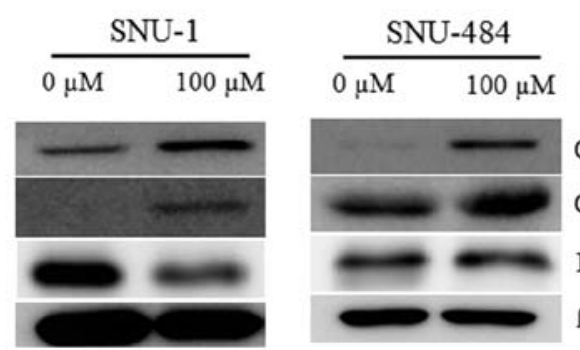

\section{Cleaved-PARP}

Cleaved-caspase-9

Pro-caspase-3

B-actin

Figure 3. Effects of DIM on caspase-mediated apoptosis in gastric cancer cell lines (SNU-1 and SNU-484). (A) Percentage of the cell population in sub-G1 phase was calculated from DNA content histograms in gastric cancer cell lines (SNU-1 and SNU-484). Error bars represent the means \pm SE from 3 independent experiments. " $\mathrm{p}<0.05$ and ${ }^{* *} \mathrm{p}<0.001$ when compared to the control. (B) Western blot analysis of cleaved-PARP, cleaved-caspase-9, and -3 following exposure of gastric cancer cells to DIM. Cells were harvested at $72 \mathrm{~h}$ and immunoblotted with the indicated antibodies. $\beta$-actin was used as an internal control. DIM, 3,3'-diindolylmethane. 

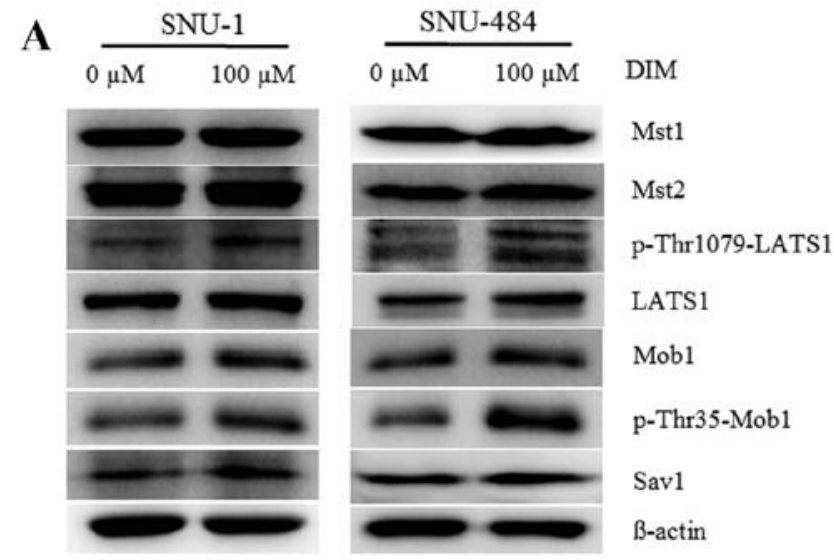

B

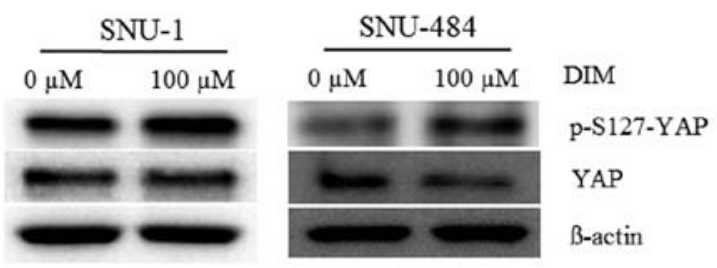

Figure 4. Effects of DIM on the Hippo signaling pathway in gastric cancer cell lines (SNU-1 and SNU-484). pYAP, YAP, Mst1/2, pLATS1, LATS1, Mob1, pMob1, and Sav1 levels were analyzed by western blotting in SNU-1 and SNU-484 cell lines after treatment with and without DIM. Cells were harvested at $72 \mathrm{~h}$, and immunoblotted with the indicated antibodies. $\beta$-actin was used as an internal control. DIM, 3,3'-diindolylmethane.

proliferation and promotes cell apoptosis. To investigate whether DIM mediates gastric cancer cell death via the Hippo signaling pathway, we examined the protein levels of Mst1/2, LATS1, pLASTS1, Mob1, pMob1, and Sav1 after DIM treatment. As shown in Fig. 4, western blot analysis revealed that treatment with $100 \mu \mathrm{M}$ DIM for $72 \mathrm{~h}$ significantly increased the production of pLATS1, pMOB1, and Sav1 in SNU-1 and SNU-484 cells. We also analyzed the production of YAP and pYAP after treatment with DIM. We found that the production of pYAP was significantly increased and YAP protein levels were decreased following treatment with $100 \mu \mathrm{M}$ DIM for $72 \mathrm{~h}$ in gastric cancer cells. These results suggest that DIM may trigger the Hippo signaling pathway cascade that leads to phosphorylation, cytoplasmic retention and functional inactivation of YAP.

DIM stimulates Mst1/2-RASSF1 interaction in gastric cancer cells. To address the question of how DIM activates the Hippo signaling pathway, we investigated the possible role of RASSF1 that has been identified as an upstream regulator of the Hippo signaling pathway $(39,40)$. RASSF1 has been reported to interact with the mammalian kinases, Mst1, and Mst2 and activate them by promoting their autophosphorylation and subsequent phosphorylation of the downstream LATS1 kinase (41). In addition, several studies have revealed that Hippo signaling is tightly regulated by protein-protein interactions (14). Therefore, we tested whether RASSF1 associates with Hippo signaling pathway molecules due to DIM treatment. As shown in Fig. 5A, endogenous RASSF1 protein production was significantly increased following treat-

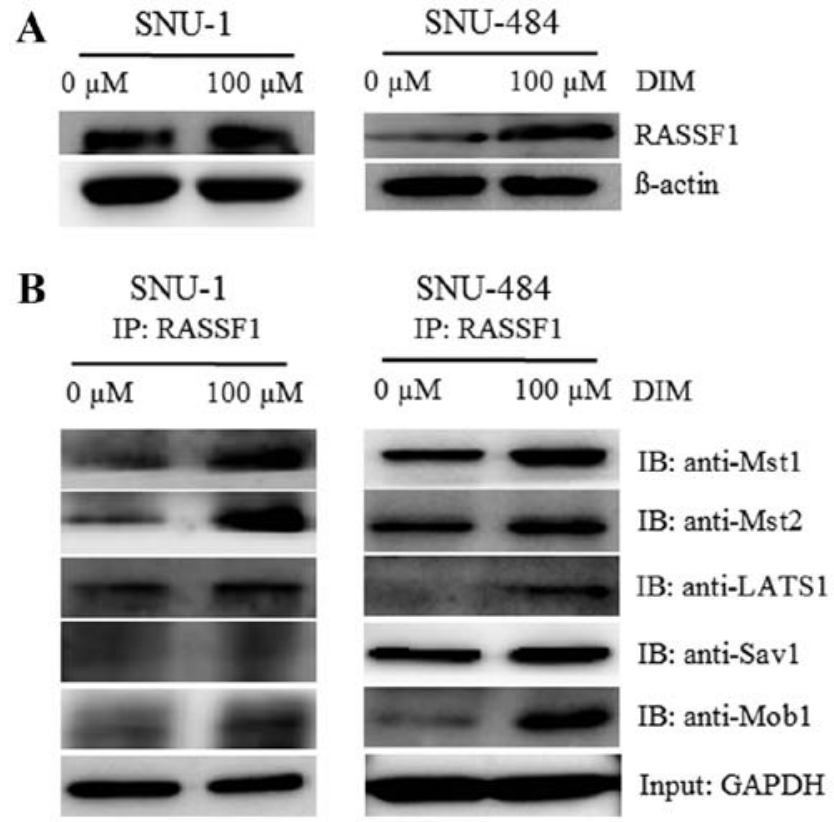

Figure 5. Effects of DIM on RASSF1 in gastric cancer cell lines (SNU-1 and SNU-484). (A) RASSF1 was analyzed by western blotting in SNU-1 and SNU-484 cells after treatment with and without DIM. Cells were harvested at $72 \mathrm{~h}$ and immunoblotted with the RASSF1 antibody. $\beta$-actin was used as an internal control. (B) Effect of DIM on the complexity of selected protein-protein interactions in the Hippo signaling pathway in gastric cancer cell lines (SNU-1 and SNU-484). SNU-1 and SNU-484 cells were either untreated or treated with DIM for $72 \mathrm{~h}$. Cells were harvested, and cell lysates were immunoprecipitated (IP) with anti-RASSF1 antibody and lysates were subjected to immunoblotting (IB) with anti-Mst1, Mst2, LATS1, Sav1, and Mob1 antibodies. GAPDH was used as an internal control. DIM, 3,3'-diindolylmethane; RASSF1, Ras association domain family 1.

ment with $100 \mu \mathrm{M}$ DIM in the SNU-1 and SNU-484 cells. In addition, a dramatic increased interaction between the Mst1/2LATS1-Sav1-Mob1 complex and RASSF1 was observed in the presence of the same DIM dose as analyzed by immunoprecipitation followed by immunoblotting (Fig. 5B). These results indicate that DIM may activate the Hippo signaling pathway through RASSF1 activation, which increases the coimmunoprecipitation of RASSF1 with Hippo signaling molecules.

DIM inhibits the growth of SNU-484 tumor xenografts in immunodeficient mice. Since we observed an inhibition of gastric cancer cell viability by DIM in vitro, we evaluated whether these observations could be translated into an animal model system in vivo. To address this issue, SPF/VAF immunodeficient mice were inoculated in the right flank with SNU-484 human gastric cancer cells. Mice were randomized into two groups and were treated daily s.c. with either vehicle or DIM $(10 \mathrm{mg} / \mathrm{kg})$ for 30 days. Tumor volume and the weight of mice were recorded once every 3 days using calipers (Fig. 6). As shown in Fig. 6A-E, DIM treatment resulted in a marked inhibition of SNU-484 xenograft tumor growth. Notably, the body weight of mice from both groups did not significantly differ from the vehicle control following 30 days of drug exposure (Fig. 6F), suggesting that DIM has no severe toxicity to the mice. Taken together, these findings demonstrate that DIM administration significantly inhibited SNU-484 xenograft growth in vivo mediated by the inactivation of YAP. 

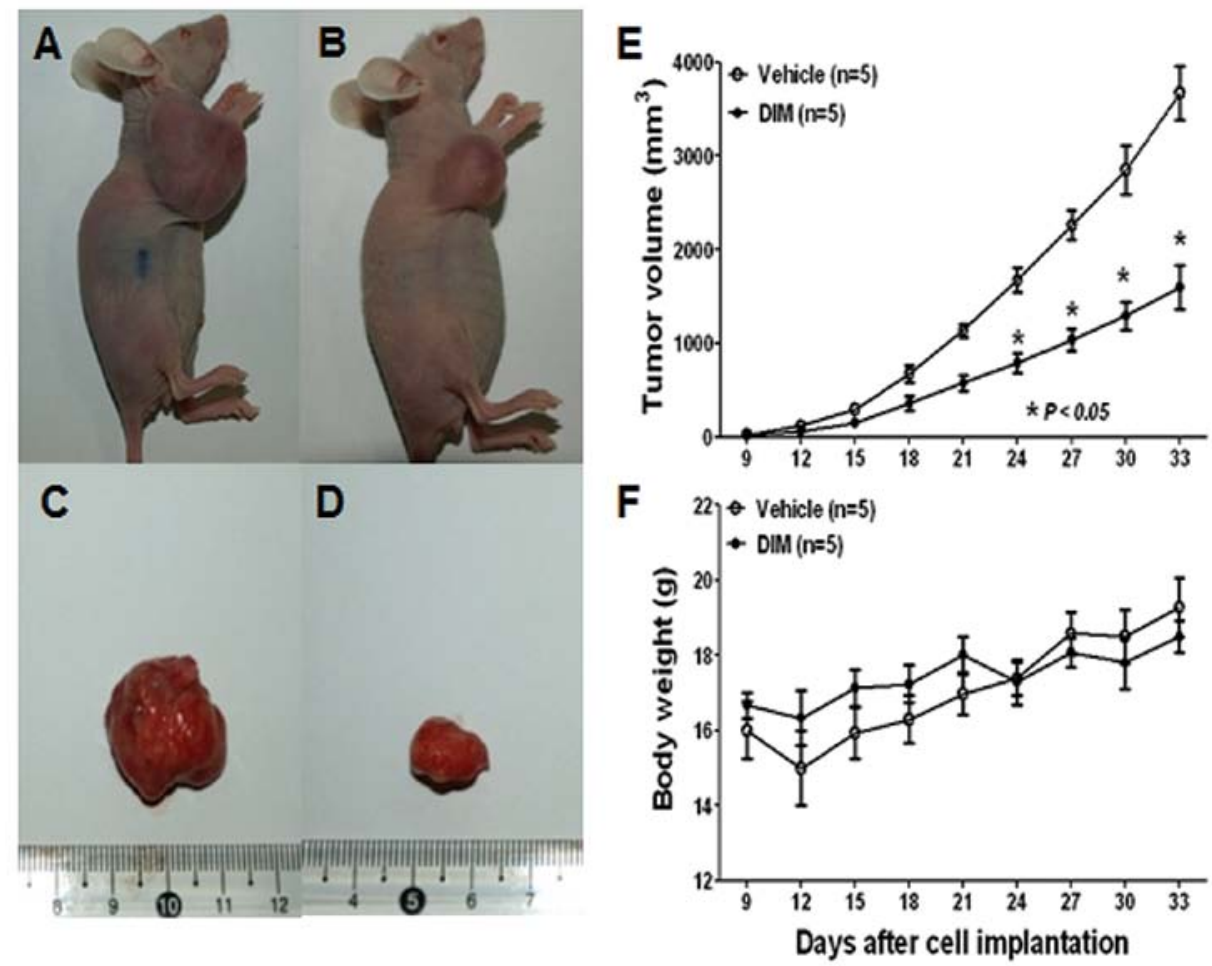

Figure 6. Effects of DIM on gastric tumor growth. DIM suppresses the growth of gastric cancer tumors in xenografted mice. Mice were inoculated subcutaneously in the right flank with $0.1 \mathrm{ml}$ Matrigel containing $7 \times 10^{6} \mathrm{SNU}-484$ human gastric cancer cells. DMSO or $10 \mathrm{mg} / \mathrm{kg}$ DIM were injected subcutaneously every day for 30 days. Tumor volume was measured twice/week using a caliper and calculated as (width) ${ }^{2} \mathrm{x}$ length/2. Representative images were captured at the end of 5 weeks of therapy, and results are shown for (A) vehicle-treated control, (B) DIM-treated mouse, (C) tumor from vehicle-treated mouse, (D) tumor from DIM-treated mouse. (E) Tumor volumes were measured after initiation of therapy. "p $<0.05$ when compared to the control. (F) Animal body weights during the course of the experiment. GAPDH was used as an internal control. DIM, 3,3'-diindolylmethane.

\section{Discussion}

The present study demonstrated for the first time that human gastric cancer cells exposed to DIM resulted in an increase in apoptosis, suggesting that DIM could be used for the treatment of gastric cancer. Despite the fact that many studies have revealed that DIM has antitumor effects in a variety of cancer cells including prostate, breast, pancreas, and esophageal cancer cells through the NF- $\mathrm{KB}$, Akt, MAPK, p53, AR, and ER pathways $(5,11,28,31,34,42)$, no information is available regarding the functional role of the Hippo signal transduction pathway in mediating DIM-induced lethality in gastric cancer cells. We found that DIM was effective in sensitizing gastric cancer cells through activation of the Hippo signaling pathway. In addition, our results provide convincing evidence in support of the antitumor effects of DIM on xenograft gastric tumors in vivo. Therefore, activation of the Hippo pathway may mediate key contributions in gastric cancer cell death induced by DIM treatment.

The evolutionarily conserved Hippo signaling pathway plays an important role in both organ size control and tumorigenesis, and thus deregulation of this pathway has been implicated in human cancers (43). Since the interruption of any factor in this pathway can bring about tumorigenesis, it appears as though YAP is the major Hippo pathway downstream effector that functions as an oncogene. In the present study, DIM dramatically increased the protein levels of pLATS1, Mob1, pMob1, and Sav1 in gastric cancer cells, while Mst1/2 levels were not altered. Intriguingly, the production of pYAP being significantly induced by DIM supports the notion that DIM activates the Hippo signaling pathway cascade leading to the inactivation of YAP function. Our observations are in agreement with previous reports demonstrating that Hippo core components can negatively regulate YAP through direct phosphorylation, and the activated LATS-Mob1 complex phosphorylates YAP leading to enhanced interactions with 14-3-3 proteins and cytoplasmic sequestration (11). Thus, the pYAP induced by DIM may limit YAP activity by preventing nuclear accumulation and decreasing the expression of positive regulators for cell growth and activators of apoptosis. In fact, we found that DIM suppressed the proliferation of various gastric cancer cell lines including SNU-1 and SNU-484 mediated by an antiproliferative effect by inducing G1 cell cycle arrest. The G1 cell cycle arrest was accompanied by the reduced production of CDK2, CDK4, CDK6 and cyclin D1 protein levels and the increased production of p53 protein levels. DIM also induced poly(ADP-ribose) polymerase cleavage, caspase-9 cleavage and diminished pro-caspase- 3 protein levels. These apoptotic effects of DIM on gastric cancer cells are in agreement with previous reports regarding other cancer cell lines $(7,28,29,37)$. Therefore, the induction of apoptosis in gastric cancer cells by DIM treatment is associated with the activation of the LATS-Mob1 complex and pYAP.

The Ras association domain family 1 (RASSF1) protein has been demonstrated to be involved in several growth regulatory and pro-apoptotic pathways. The RASSF1 gene 
is localized on chromosome $3 \mathrm{p} 21.3$ and is found to be frequently silenced by promoter hypermethylation in a variety of cancers $(39,40,44,45)$. Targeted disruption of the RASSF1 gene in mice resulted in spontaneous and carcinogen-induced tumorigenesis (46), indicating that RASSF1 serves as an important tumor-suppressor. Recent research has shown that Hippo signal transduction pathway activity is regulated by RASSF1, an emerging pathway implicated in the control of cell growth, apoptosis, and tumor-suppression $(20,41,47)$. In addition, several studies have shown that RASSF1 interacts with the mammalian Mst1/2, and promotes phosphorylation of Mst1/2 and their downstream gene products $(48,49)$, suggesting that RASSF1-Mst1/2 may have intriguing tumor-suppressing characteristics. In fact, a recent study revealed that RASSF1 prevented dephosphorylation of Mst1/2 by binding to Mst1/2, supporting the maintenance of pMst1/2 phosphorylation (41). In the present study, we found that endogenous RASSF1 protein levels were significantly increased by DIM treatment in gastric cancer cells. The binding of Mst1/2, LAST1 and Mob1 increased when interacting with RASSF1, suggesting that DIM stimulates RASSF1 with the Mst1/2-LAST-Mob complex to promote an active state of the Mst kinases, favoring the induction of pYAP that inactivates cell proliferation. Therefore, these results indicate that RASSF1 interacts with Mst1/2 by DIM treatment and the cytotoxic effects of DIM on gastric cancer cells are mediated through the Hippo signaling pathway.

Our in vitro results were recapitulated in vivo using a gastric tumor xenograft animal model. We found that DIM significantly inhibited the growth of gastric tumors. Particularly, an increase in pYAP in the DIM-treated tumors was observed. These in vivo results were consistent with our molecular studies in vitro, which obviously provide strong support that Hippo signaling and the YAP pathway play a significant role in gastric cancer cell proliferation and may therefore be potential targets for the treatment of gastric cancer. Therefore, our study suggests that DIM acts as a promising agent against gastric tumors in vivo as it not only reduced tumor growth but also suppressed YAP activation that strongly correlated with the stimulation of tumor growth.

In conclusion, our study provided strong evidence that DIM induces human gastric cancer cell death that is due in part to activation of the Hippo signal transduction pathway and the inactivation of YAP. Our in vitro findings together with our in vivo results provide important implications of DIM as a chemopreventive or therapeutic agent for improving the outcome of gastric cancer patients.

\section{Acknowledgements}

This study was supported by the Bumsuk Academic Research Fund in 2011.

\section{References}

1. Kong D, Banerjee S, Huang W, et al: Mammalian target of rapamycin repression by 3,3'-diindolylmethane inhibits invasion and angiogenesis in platelet-derived growth factor-D-overexpressing PC3 cells. Cancer Res 68: 1927-1934, 2008.

2. Shin HR, Carlos MC and Varghese C: Cancer control in the Asia Pacific region: current status and concerns. Jpn J Clin Oncol 42 867-881, 2012.
3. Parkin DM, Bray F, Ferlay J and Pisani P: Global cancer statistics, 2002. CA Cancer J Clin 55: 74-108, 2005.

4. Tan IB, Ng I, Tai WM and Tan P: Understanding the genetic basis of gastric cancer: recent advances. Expert Rev Gastroenterol Hepatol 6: 335-341, 2012.

5. Hoffmann W: Stem cells, self-renewal and cancer of the gastric epithelium. Curr Med Chem 19: 5975-5983, 2012.

6. Cho JY, Lim JY, Cheong JH, et al: Gene expression signaturebased prognostic risk score in gastric cancer. Clin Cancer Res 17: 1850-1857, 2011.

7. Hundahl SA, Phillips JL and Menck HR: The National Cancer Data Base Report on poor survival of U.S. gastric carcinoma patients treated with gastrectomy: Fifth Edition American Joint Committee on Cancer staging, proximal disease, and the 'different disease' hypothesis. Cancer 88: 921-932, 2000.

8. Kovoor PA and Hwang J: Treatment of resectable gastric cancer: current standards of care. Exp Rev Anticancer Ther 9: 135-142, 2009.

9. Rausei S, Dionigi G, Rovera F, et al: A decade in gastric cancer curative surgery: evidence of progress (1999-2009). World J Gastrointest Surg 4: 45-54, 2012.

10. Harvey KF, Pfleger CM and Hariharan IK: The Drosophila Mst ortholog, hippo, restricts growth and cell proliferation and promotes apoptosis. Cell 114: 457-467, 2003.

11. Harvey K and Tapon N: The Salvador-Warts-Hippo pathway an emerging tumour-suppressor network. Nat Rev Cancer 7: 182-191, 2007.

12. Grusche FA, Richardson HE and Harvey KF: Upstream regulation of the hippo size control pathway. Curr Biol 20: R574-R582, 2010.

13. Harvey KF and Hariharan IK: The hippo pathway. Cold Spring Harb Perspect Biol 4: a011288, 2012.

14. Zeng Q and Hong W: The emerging role of the hippo pathway in cell contact inhibition, organ size control, and cancer development in mammals. Cancer Cell 13: 188-192, 2008.

15. Edgar BA: From cell structure to transcription: Hippo forges a new path. Cell 124: 267-273, 2006

16. Zhao B, Li L, Lei Q and Guan KL: The Hippo-YAP pathway in organ size control and tumorigenesis: an updated version. Genes Dev 24: 862-874, 2010.

17. Xu ZP, Zhu JS, Zhang Q and Wang XY: A breakdown of the Hippo pathway in gastric cancer. Hepatogastroenterology 58: 1611-1617, 2011.

18. Zhou Z, Zhu JS, Xu ZP and Zhang Q: Lentiviral vector-mediated siRNA knockdown of the YAP gene inhibits growth and induces apoptosis in the SGC7901 gastriccancer cell line. Mol Med Rep 4: 1075-1082, 2011.

19. Min B, Kim MK, Zhang JW, et al: Identification of RUNX3 as a component of the MST/Hpo signaling pathway. J Cell Physiol 227: 839-849, 2012.

20. Yoshihama Y, Izumisawa Y, Akimoto K, et al: High expression of KIBRA in low atypical protein kinase C-expressing gastric cancer correlates with lymphatic invasion and poor prognosis. Cancer Sci 104: 259-265, 2013.

21. Song M, Cheong JH, Kim H, Noh SH and Kim H: Nuclear expression of Yes-associated protein 1 correlates with poor prognosis in intestinal type gastric cancer. Anticancer Res 32: 3827-3834, 2012

22. Lam-Himlin DM, Daniels JA, Gayyed MF, et al: The hippo pathway in human upper gastrointestinal dysplasia and carcinoma: a novel oncogenic pathway. Int J Gastrointest Cancer 37: 103-109, 2006.

23. Seymour JD, Calle EE, Flagg EW, Coates RJ, Ford ES and Thun MJ; American Cancer Society: Diet Quality Index as a predictor of short-term mortality in the American Cancer Society Cancer Prevention Study II Nutrition Cohort. Am J Epidemiol 157: 980-988, 2003.

24. Gao N, Cheng S, Budhraja A, et al: 3,3'-Diindolylmethane exhibits antileukemic activity in vitro and in vivo through a Akt-dependent process. PloS One 7: e31783, 2012.

25. Grose KR and Bjeldanes LF: Oligomerization of indole-3-carbinol in aqueous acid. Chem Res Toxicol 5: 188-193, 1992.

26. Keck AS and Finley JW: Cruciferous vegetables: cancer protective mechanisms of glucosinolate hydrolysis products and selenium. Integr Cancer Ther 3: 5-12, 2004.

27. Banerjee S, Wang Z, Kong D and Sarkar FH: 3,3'-Diindolylmethane enhances chemosensitivity of multiple chemotherapeutic agents in pancreatic cancer. Cancer Res 69: 5592-5600, 2009. 
28. Kim SJ, Lee JS and Kim SM: 3,3'-Diindolylmethane suppresses growth of human esophageal squamous cancer cells by G1 cell cycle arrest. Oncol Rep 27: 1669-1673, 2012.

29. Ali S, Banerjee S, Ahmad A, El-Rayes BF, Philip PA and Sarkar FH: Apoptosis-inducing effect of erlotinib is potentiated by 3,3'-diindolylmethane in vitro and in vivo using an orthotopic model of pancreatic cancer. Mol Cancer Ther 7: 1708-1719, 2008.

30. Chinnakannu K, Chen D, Li Y, et al: Cell cycle-dependent effects of 3,3'-diindolylmethane on proliferation and apoptosis of prostate cancer cells. J Cell Physiol 219: 94-99, 2009.

31. Bhuiyan MM, Li Y, Banerjee S, et al: Down-regulation of androgen receptor by 3,3'-diindolylmethane contributes to inhibition of cell proliferation and induction of apoptosis in both hormone-sensitive $\mathrm{LNCaP}$ and insensitive $\mathrm{C} 4-2 \mathrm{~B}$ prostate cancer cells. Cancer Res 66: 10064-10072, 2006.

32. Li Y, Chinni SR and Sarkar FH: Selective growth regulatory and pro-apoptotic effects of DIM is mediated by AKT and NF-kappaB pathways in prostate cancer cells. Front Biosci 10: 236-243, 2005.

33. Nachshon-Kedmi M, Fares FA and Yannai S: Therapeutic activity of 3,3'-diindolylmethane on prostate cancer in an in vivo model. Prostate 61: 153-160, 2004.

34. Rahman KW and Sarkar FH: Inhibition of nuclear translocation of nuclear factor- $\kappa \mathrm{B}$ contributes to 3,3'-diindolylmethane-induced apoptosis in breast cancer cells. Cancer Res 65: 364-371, 2005.

35. Abdelrahim M, Newman K, Vanderlaag K, Samudio I and Safe S: 3,3'-diindolylmethane (DIM) and its derivatives induce apoptosis in pancreatic cancer cells through endoplasmic reticulum stress-dependent upregulation of DR5. Carcinogenesis 27: 717-728, 2006

36. Carter TH, Liu K, Ralph W Jr, et al: Diindolylmethane alters gene expression in human keratinocytes in vitro. J Nutr 132: 3314-3324, 2002.

37. Azmi AS,Ahmad A,Banerjee S, Rangnekar VM, Mohammad RM and Sarkar FH: Chemoprevention of pancreatic cancer: characterization of Par-4 and its modulation by 3,3' diindolylmethane (DIM). Pharm Res 25: 2117-2124, 2008.

38. Kim SJ, Chung MJ, Kim JS, et al: Deciphering the role of paclitaxel in the SKGT4 human esophageal adenocarcinoma cell line. Int J Oncol 39: 1587-1591, 2011.
39. Dammann R, Li C, Yoon JH, Chin PL, Bates S and Pfeifer GP: Epigenetic inactivation of a RAS association domain family protein from the lung tumour suppressor locus 3p21.3. Nat Genet 25: 315-319, 2000.

40. Dammann R, Schagdarsurengin U, Seidel C, et al: The tumor suppressor RASSF1A in human carcinogenesis: an update. Histol Histopathol 20: 645-663, 2005.

41. Guo C, Zhang X and Pfeifer GP: The tumor suppressor RASSF1A prevents dephosphorylation of the mammalian STE20-like kinases MST1 and MST2. J Biol Chem 286: 6253-6261, 2011.

42. Chen Y, Xu J, Jhala N, et al: Fas-mediated apoptosis in cholangiocarcinoma cells is enhanced by 3,3'-diindolylmethane through inhibition of AKT signaling and FLICE-like inhibitory protein. Am J Pathol 169: 1833-1842, 2006.

43. Saucedo LJ and Edgar BA: Filling out the Hippo pathway. Nat Rev Mol Cell Biol 8: 613-621, 2007.

44. Burbee DG, Forgacs E, Zöchbauer-Müller S, et al: Epigenetic inactivation of RASSF1A in lung and breast cancers and malignant phenotype suppression. J Natl Cancer Inst 93: 691-699, 2001.

45. Agathanggelou A, Cooper WN and Latif F: Role of the Ras-association domain family 1 tumor suppressor gene in human cancers. Cancer Res 65: 3497-3508, 2005.

46. Tommasi S, Dammann R, Zhang Z, et al: Tumor susceptibility of Rassf 1a knockout mice. Cancer Res 65: 92-98, 2005.

47. Vichalkovski A, Gresko E, Cornils H, Hergovich A, Schmitz D and Hemmings BA: NDR kinase is activated by RASSF1A/MST1 in response to Fas receptor stimulation and promotes apoptosis. Curr Biol 18: 1889-1895, 2008.

48. Guo C, Tommasi S, Liu L, Yee JK, Dammann R and Pfeifer GP: RASSF1A is part of a complex similar to the Drosophila Hippo/Salvador/Lats tumor-suppressor network. Curr Biol 17: 700-705, 2007

49. Matallanas D, Romano D, Yee K, et al: RASSF1A elicits apoptosis through an MST2 pathway directing proapoptotic transcription by the p73 tumor suppressor protein. Mol Cell 27: 962-975, 2007. 